Im untenstehenden Artikel werden von Seiten des BAG nochmals sehr übersichtlich und gut verständlich die Voraussetzungen hinsichtlich Aus- und Weiterbildung in Erinnerung gerufen, die zur Durchführung von konventionellen Aufnahmen im mittleren Dosisbereich berechtigen. Diese Informationen betreffen die Ärzteschaft und die Medizinischen Praxisassistentinnen und -assistenten EFZ. Alle Kolleginnen und Kollegen, welche konventionelle Aufnahmen im mittleren Dosisbereich selber durchführen oder durch MPA EFZ durchführen lassen, bitten wir, diese Informationen des BAG genau zu studieren.

\title{
Konventionelle Röntgenaufnahmen im mittleren Dosisbereich
}

\section{Nicolas Stritt ${ }^{a}$, Thomas Flury ${ }^{b}$, Annemarie Harwig ${ }^{c}$}

${ }^{a}$ Dr. phil. nat., Leiter der Sektion Forschungsanlagen und Nuklearmedizin; ${ }^{b}$ Dr. phil. nat., Wissenschaftlicher Mitarbeiter; ${ }^{c}$ Wissenschaftliche Mitarbeiterin

Die Bedienung von medizinischen Röntgengeräten ist den Berufsgruppen vorbehalten, welche gemäss Strahlenschutzverordnung über die entsprechende und anerkannte Ausbildung im Strahlenschutz verfügen. Für die Ausbildung zum Erwerb der notwendigen Kompetenzen wird unterschieden, ob es sich bei den Röntgenaufnahmen um Aufnahmen aus dem Niedrigdosisbereich, mittleren Dosisbereich oder Hochdosisbereich handelt. Dieser Artikel informiert über die bevorstehende Umfrage des BAG und gibt einen Überblick über die notwendigen Ausbildungen beziehungsweise Kompetenzen, welche Ärztinnen/Ärzte und das medizinische Personal im Bereich der konventionellen Diagnostik besitzen müssen. Detaillierte Informationen über die Umfrage des BAG und die notwendigen Aus- und Weiterbildungen finden sich unter: www.bag.admin.ch/roentgen-arztpraxen

\section{Audit in den Arztpraxen}

Die Abteilung Strahlenschutz des Bundesamts für Gesundheit BAG hat vor einigen Jahren circa 100 Betriebe auditiert [1]. Dabei bot sich die Gelegenheit, die Anwenderinnen und Anwender bezüglich wichtiger Strahlenschutzaspekte und erforderlicher Ausbildung zu sensibilisieren. Die Analyse der Erhebungen zeigte sowohl bei den Ärzten wie auch bei den Medizinischen Praxisassistentinnen/Praxisassistenten (MPA) einen Nachholbedarf im Bereich der notwendigen Ausbildung im Strahlenschutz zur Anfertigung von Aufnahmen im mittleren Dosisbereich (konventionell-radiologische Untersuchungen des Abdomens, Achsenskeletts und Beckens). Bei den MPA wurde festgestellt, dass in über 80\% der Fälle die erforderliche Ausbildung für die erweiterten konventionellen Aufnahmetechniken nicht absolviert worden ist, obwohl sie solche Aufnahmen durchführen.

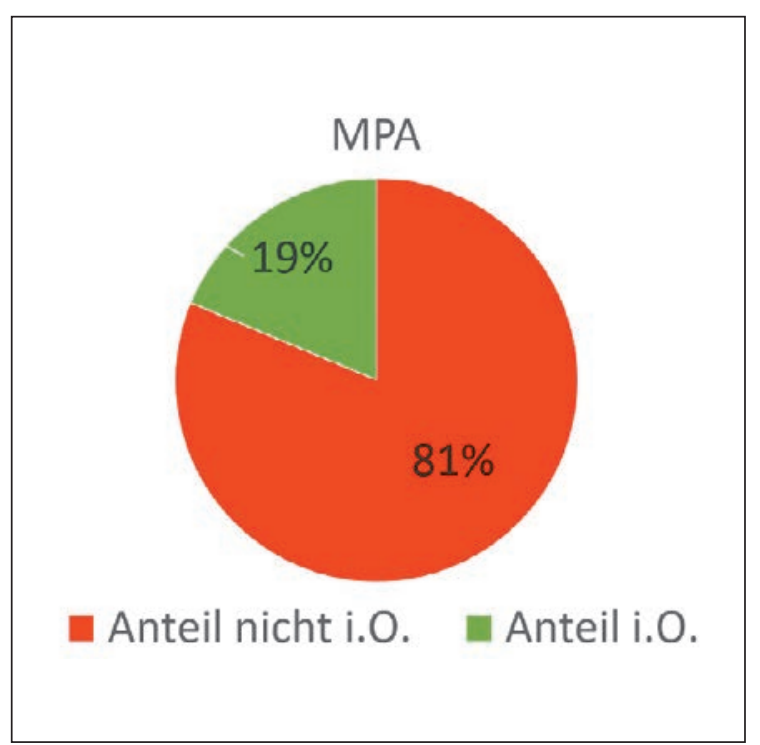

Abbildung 1: Ausbildungsstand erweiterte konventionelle Aufnahmetechniken in den auditierten Arztpraxen. 
Das Absolvieren einer entsprechenden, anerkannten Ausbildung ist Garant, dass das Personal mit den notwendigen Kompetenzen zur Erreichung eines effektiven Strahlenschutzes ausgerüstet wird. Dies ermöglicht eine Optimierung der Strahlendosen für die Patienten. Zum Schutz der Patienten ist es deshalb wichtig, dass in der Praxis jede Ärztin/jeder Arzt, die/ der Aufnahmen im mittleren Dosisbereich durchführt und beurteilt, in Besitz des entsprechenden Fähigkeitsausweises «Dosisintensives Röntgen (KHM)» [2] ist und mindestens ein/e MPA mit der Weiterbildung «Erweiterte konventionelle Aufnahmetechniken", besser bekannt unter dem Namen «Dosisintensives Röntgen», in der Praxis tätig ist und die Aufnahmen durchführt.

\section{Weiterbildung «Erweiterte konven- tionelle Aufnahmetechniken"}

MPA erlangen in der beruflichen Grundausbildung (3 Jahre) die Berechtigung für die Bedienung von Röntgenanlagen im Niedrigdosisbereich (Thorax und Extremitäten) nach Anweisung einer entsprechenden sachverständigen Ärztin oder eines entsprechenden sachverständigen Arztes.

Die zusätzliche Strahlenschutzweiterbildung für die Durchführung von Röntgenaufnahmen im mittleren Dosisbereich gab es bereits und ist keine Neuerung der revidierten Strahlenschutzverordnung [3] und der Strahlenschutz-Ausbildungsverordnung [4], welche am 1. Januar 2018 in Kraft getreten ist.

Während der 40 Unterrichtseinheiten der Weiterbildung werden die Teilnehmerinnen und Teilnehmer

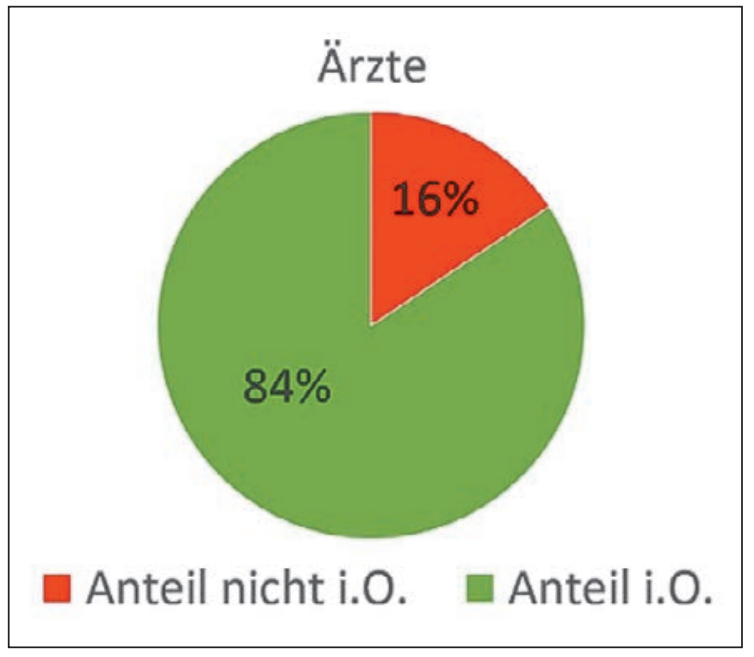

Abbildung 2: Ausbildungsstand der Ärzte in den auditierten Arztpraxen.

mit den Einstelltechniken in den Bereichen Abdomen, Achsenskelett und Schädel vertraut gemacht und lernen die Röntgenbilder bezüglich Bildparameter, Kontrast und Schärfe zu interpretieren. Nach erfolgreichem Abschluss sind die MPA im Besitz der notwendigen Ausbildung im Strahlenschutz für Röntgenaufnahmen im mittleren Dosisbereich und Niedrigdosisbereich nach Anweisung einer entsprechenden sachverständigen Ärztin oder eines entsprechenden sachverständigen Arztes. Jedoch ist es einer/einem MPA auch mit dieser zusätzlichen Weiterbildung nicht erlaubt, CT-Untersuchungen, Untersuchungen mit durchleuchtungsgestützten Anlagen und die Mammographien durchzuführen.

Tabelle 1: Erlaubte und nicht erlaubte Tätigkeiten für das medizinische Personal im Bereich Röntgen als Funktion der beruflichen Aus- und Weiterbildung.

\begin{tabular}{|c|c|c|}
\hline \multicolumn{3}{|c|}{ Konventionelles Projektionsröntgen } \\
\hline $\begin{array}{l}\text { Berufsgruppe } \\
\text { (Abkürzung aus der Ausbildungs- } \\
\text { verordnung) }\end{array}$ & $\begin{array}{l}\text { Niedrigdosisbereich: } \\
\text { Thorax und Extremitäten }\end{array}$ & $\begin{array}{l}\text { Mittlerer Dosisbereich: } \\
\text { Abdomen, Wirbelsäule, Schädel }\end{array}$ \\
\hline $\begin{array}{l}\text { MP7 } \\
\text { MPA mit EFZ } \\
\text { MP9 } \\
\text { Übriges med. Personal mit vom BAG aner- } \\
\text { kannter Röntgenberechtigung Thorax und } \\
\text { Extremitäten }\end{array}$ & $\begin{array}{c}\sqrt{ } \\
\text { Bedienung der Anlage nach Anweisung } \\
\text { einer sachverständigen Ärztin/eines } \\
\text { sachverständigen Arztes }\end{array}$ & $\begin{array}{c}\mathbf{x} \\
\text { Ohne Weiterbildung «Erweiterte } \\
\text { konventionelle Aufnahmetechniken» } \\
\text { nicht erlaubt }\end{array}$ \\
\hline $\begin{array}{l}\text { MP8 } \\
\text { MPA mit EFZ und einer Weiterbildung } \\
\text { «Erweiterte konventionelle Aufnahme- } \\
\text { techniken» } \\
\text { Übriges med. Personal mit Röntgenberech- } \\
\text { tigung Thorax und Extremitäten und einer } \\
\text { Weiterbildung "Erweiterte konventionelle } \\
\text { Aufnahmetechniken» }\end{array}$ & $\begin{array}{c}\sqrt{ } \\
\text { Bedienung der Anlage nach Anweisung } \\
\text { einer sachverständigen Ärztin/eines } \\
\text { sachverständigen Arztes }\end{array}$ & $\begin{array}{c}\sqrt{ } \\
\text { Bedienung der Anlage nach Anweisung } \\
\text { einer sachverständigen Ärztin/eines } \\
\text { sachverständigen Arztes }\end{array}$ \\
\hline $\begin{array}{l}\text { MP4/6 } \\
\text { Dipl. Radiologiefachpersonen HF/FH (MTRA) }\end{array}$ & $\begin{array}{l}\sqrt{ } \\
\text { Bedienung der Anlage nach Anweisung } \\
\text { einer sachverständigen Ärztin/eines } \\
\text { sachverständigen Arztes }\end{array}$ & $\begin{array}{c}\sqrt{ } \\
\text { Bedienung der Anlage nach Anweisung } \\
\text { einer sachverständigen Ärztin/eines } \\
\text { sachverständigen Arztes }\end{array}$ \\
\hline
\end{tabular}




\begin{tabular}{|c|c|c|}
\hline & Konventionelles Projektionsröntgen & \\
\hline $\begin{array}{l}\text { MA } 11 \\
-\quad \text { Alle Ärztinnen und Ärzte }\end{array}$ & $\begin{array}{c}\sqrt{ } \\
\text { Rechtfertigen, } \\
\text { Durchführen und Befunden } \\
\text { der konventionellen Aufnahmen }\end{array}$ & $\begin{array}{c}\mathbf{x} \\
\text { Ohne Fähigkeitsausweis } \\
\text { «Dosisintensives Röntgen» } \\
\text { nicht erlaubt }\end{array}$ \\
\hline $\begin{array}{l}\text { MA } 6 \\
\text { - } \text { Allgemeine Innere Medizin } \\
\text { - Kinder- und Jugendmedizin } \\
-\quad \text { Medizinische Onkologie } \\
\text { - Neurologie } \\
-\quad \text { Praktische Ärztin/praktischer Arzt } \\
\text { - } \quad \text { Rheumatologie } \\
\text { Zusätzlich mit } \\
-\quad \text { Fähigkeitsausweis «Dosisintensives } \\
\quad \text { Röntgen (KHM)» (www.kollegium.ch) }\end{array}$ & $\begin{array}{c}\sqrt{ } \\
\text { Rechtfertigen, } \\
\text { Durchführen und Befunden } \\
\text { der konventionellen Aufnahmen }\end{array}$ & $\begin{array}{c}\sqrt{ } \\
\text { Rechtfertigen, } \\
\text { Durchführen und Befunden } \\
\text { der konventionellen Aufnahmen }\end{array}$ \\
\hline $\begin{array}{l}\text { MA } 5 \\
\text { - Chirurgie } \\
-\quad \text { Orthopädische Chirurgie und Traumato- } \\
\quad \text { logie des Bewegungsapparates }\end{array}$ & $\begin{array}{c}\sqrt{ } \\
\text { Rechtfertigen, } \\
\text { Durchführen und Befunden } \\
\text { der konventionellen Aufnahmen }\end{array}$ & $\begin{array}{c}\sqrt{ } \\
\text { Rechtfertigen, } \\
\text { Durchführen und Befunden } \\
\text { der konventionellen Aufnahmen }\end{array}$ \\
\hline
\end{tabular}

\section{Fähigkeitsausweis «Dosisintensives Röntgen (KHM)»}

Das Audit zeigte, dass bei den Ärztinnen/Ärzten in rund 20\% die gesetzlich erforderliche Weiterbildung nicht absolviert worden ist.

Für den Erhalt der entsprechenden Kompetenzen für Aufnahmen im mittleren Dosisbereich sind für die einzelnen Weiterbildungstitel die in Tabelle 2 dargelegten zusätzlichen Weiterbildungen im Strahlenschutz notwendig.

Die Bewilligungsinhaberin, der Bewilligungsinhaber trägt die Verantwortung, dass für die Durchführung von Röntgenaufnahmen entsprechend qualifiziertes Personal (Ärzte und medizinisches Personal) eingesetzt wird und insbesondere bei den erweiterten konventionellen Aufnahmetechniken darauf geachtet wird, dass die zusätzlichen Kompetenzen vorhanden sind.

\section{Online-Umfrage zur Evaluation des jetzigen Ausbildungsstands in den Arztpraxen}

Als Massnahme hat das BAG vor einigen Jahren alle gut 3500 Arztpraxen, welche im Besitz einer Röntgenanlage sind, angeschrieben und darauf hingewiesen, dass dieses Ausbildungsmanko zu beheben ist. Das BAG, Abteilung Strahlenschutz, möchte evaluieren, inwiefern sich die Situation seither verbessert hat und wo noch angesetzt werden muss.

Die Abteilung Strahlenschutz des BAG wird gestaffelt in den kommenden Monaten jeder Praxis mit einer
Röntgenanlage eine schriftliche Aufforderung zur Online-Umfrage zukommen lassen. Die Umfrage soll über folgende Aspekte Informationen liefern:

- Ausbildungsstand allfälliger Ärzte, die Befunde und/oder Röntgenaufnahmen anfertigen.

- Ausbildungsstand des Personals, welche die Röntgenanlage im Niedrigdosisbereich (Thorax und Extremitäten) und mittleren Dosisbereich (Abdomen, Achsenskelett und Becken) bedient.

- Statistik der Anzahl durchgeführter Röntgenbilder pro Monat.

\section{Informationen}

Das BAG hat auf seiner Internetseite www.bag.admin. ch/roentgen-arztpraxen Informationen über die Umfrage, die notwendige Ausbildung, Weiterbildung und die erlaubten Tätigkeiten im Strahlenschutz veröffentlicht.

Literatur

1 Schlussbericht zum Aufsichtsschwerpunkt: digitale Radiologie in Arztpraxen; https://www.bag.admin.ch/bag/de/home/gesetzeund-bewilligungen/gesuche-bewilligungen/bewilligungen-aufsicht-im-strahlenschutz/schlussberichte-aufsichtsschwerpunkte. html

2 Einzelheiten zum Fähigkeitsausweis finden sich unter www.kollegium.ch

3 Einzelheiten zur Strahlenschutzverordnung (StSV) finden sich unter https://www.admin.ch/opc/de/classified-compilation/ 20163016/index.html.

4 Einzelheiten zur Strahlenschutz-Ausbildungsverordnung (StAV) finden sich unter https://www.admin.ch/opc/de/classifiedcompilation/20163019/index.html. 